\title{
Cognitive control functions in unipolar major depression with and without co-morbid anxiety disorder
}

\author{
Pia Lyche ${ }^{1 *}$, Rune Jonassen ${ }^{1,2}$, Tore C. Stiles ${ }^{3}$, Pål Ulleberg ${ }^{1}$ and Nils I. Landrø ${ }^{1}$ \\ 'Department of Psychology, Center for the Study of Human Cognition, University of Oslo, Oslo, Norway \\ 2 Akershus University Hospital Health Authority, Lørenskog, Norway \\ ${ }^{3}$ Department of Psychology, Norwegian University of Science and Technology, Trondheim, Norway
}

Edited by:

Mark A. Frye, Mayo Clinic, USA

Reviewed by:

Robert H. Howland, University of

Pittsburgh School of Medicine, USA

Mark A. Frye, Mayo Clinic, USA

*Correspondence:

Pia Lyche, Department of Psychology, University of Oslo, P.O. Box 1094,

Blindern, NO-0317 Oslo, Norway.

e-mail: p.e.lyche@psykologi.uio.no

\begin{abstract}
Background: Impaired cognitive control functions have been demonstrated in both major depression (MDD) and anxiety disorder (A), but few studies have systematically examined the impact of MDD with co-morbid A (MDDA), which is the main aim of this study. Method: We compared patients with MDD with (MDDA; $n=24$ ) and without co-morbid A $(n=37)$ to a group of healthy controls ( $\mathrm{HC} ; n=92)$ on three subtests from the Cambridge NeuropsychologicalTest Automated Battery; intra-extra dimensional, stop signal task, and spatial working memory. These tasks correspond to a theoretical model consisting of three separable but interrelated executive control functions: Shifting, Inhibition, and Updating. A simple psychomotor speed measure was also included. Results: After controlling for age, gender, and education level, the results showed that the MDDA group displayed significantly impaired performance on the functions Shifting and Updating compared to $\mathrm{HC}$. There emerged no significant differences between any of the patient groups and $\mathrm{HC}$ regarding Inhibition. The pure MDD group did not display dysfunctions relative to the $\mathrm{HC}$ group on the main executive control variables, but displayed slowed psychomotor speed. Contrary to expectation there were no significant differences between the MDDA and the MDD groups. Conclusion: Co-morbid anxiety should be taken into account when studying cognitive control functions in major depression.
\end{abstract}

Keywords: unipolar major depression, co-morbid anxiety disorder, cognitive control functions, CANTAB

\section{INTRODUCTION}

Depressive disorders present a significant mental health concern to individuals and to our society. It is estimated that mood disorders will be the most frequent cause of serious health problems worldwide in 2020 (WHO). The lifetime risk for major depressive disorder (MDD) is between 10 and 25\% for women and between 5 and 12\% for men (American Psychiatric Association, 1994).

Affective neuroscience research has been increasingly focusing on cognitive or executive control processes (for a review, see Ochsner and Gross, 2005). Investigations of executive control functions are in general relevant across a range of psychiatric disorders, like schizophrenia (Wilmsmeier et al., 2010), obsessive compulsive disorder (Olley et al., 2007), and bipolar disorder (Daban et al., 2006). Although these disorders share a common deficit in executive functions, a main issue is to what degree there are differences and similarities along various dimensions of executive functions across such disorders.

Retarded psychomotor speed, as measured by simple reaction time tests, was traditionally considered a basic clinical characteristic of unipolar MDD. Although slowed reactions obviously are a relevant aspect of the neuropsychological profile in depression (Egeland et al., 2005), associated cognitive dysfunctions cover a broad range (Burt et al., 1995; Christensen et al., 1997; Kindermann and Brown, 1997; Veiel, 1997; Austin et al., 2001; Landrø et al., 2001; Porter et al., 2007). Impaired executive cognitive control functions seems to be particularly salient (Austin et al., 2001; Rogers et al.,
2004; Levin et al., 2007; Porter et al., 2007 for reviews). The few studies distinguishing between unipolar and bipolar depressed patients have not displayed a consistent picture (Gruber et al., 2007 ) but impaired inhibitory control seem to be more pronounced in bipolar versus MDD patients.

Findings link cognitive control deficits in MDD to increase in negative affect (McNeely et al., 2005; Dennis and Chen, 2007), a tendency to ruminate (Nolen-Hoeksema, 1991), increasing number of depressive episodes (Vanderhasselt and De Raedt, 2009), and a family history of depression in children (Perez-Edgar et al., 2006; Vanderhasselt and De Raedt, 2009).

Cognitive control embodies the ability to organize incoming stimuli and inhibit a dominant response in order to perform a subdominant response (MacLeod et al., 2002). However, executive functions are complex, and because they manifest themselves by operating on other cognitive processes, this implicates other cognitive processes not directly relevant to the target executive function, hence "the task impurity problem" (Philips, 1997). Executive tasks have also tended to suffer from relatively low internal and/or testretest reliability (Denckla, 1996; Rabbit, 1997; Miyake et al., 2000), and widely used and accepted tests like, i.e., WCST and TOH have based their construct validity on loose criteria such as "sensitive to frontal lobe damage." The complexity of the construct is also reflected in the myriad of terms and definitions used to characterize executive control functions in addition to the tests used to measure them. 
In an attempt to minimize the task impurity problem, Miyake et al. (2000) used latent variable analysis to determine to what extent different executive processes can be considered to be unitary (in the sense that they are reflections of the same underlying mechanism or ability) or non-unitary. They focused on the following three basic executive functions: (1) shifting between tasks or mental sets, (2) inhibition of dominant or prepotent responses, and (3) updating and monitoring the contents of working memory. Confirmatory factor analysis indicated that these three executive processes, although moderately correlated with one another, are clearly separable. The model proposes relatively circumscribed lower (basic) level functions compared to many of the wider and higher level definitions and general measures of executive functioning, thus allowing precision as to which cognitive control functions are affected and which are not. However, neuropsychological studies of mood disorders based on an empirically based model of basic executive control functions are relatively sparse.

There is extensive co-morbidity between MDD and other affective disorders, especially anxiety disorders (A), regarding phenomenological features as well as neuropsychological functioning. Individuals with depression and co-morbid anxiety disorders occur in from 25 to $60 \%$ of cases, and are associated with increased severity, greater chronicity, slower recovery, increased rates of recurrence, greater risk for suicide, and greater psychosocial disability (Cameron et al., 2004; Leonardo and Hen, 2006).

Anxiety can potentially confound or contribute to neuropsychological impairments or anatomic changes in depressed individuals (Cameron et al., 2004; Leonardo and Hen, 2006), and is in general associated with heightened distractibility, poor concentration, and increased responsivity to potential threat (Bishop et al., 2004b). Several studies have demonstrated neuropsychological deficits involving, i.e., executive function, attention, working memory, and attentional control to threat-related stimuli in anxiety disorders (Broadbent et al., 1986; Asmundson et al., 1994; Cohen et al., 1996; Vasterling et al., 2000; Lautenbacher et al., 2002; Danckwerts and Leathem, 2003; Ludewig et al., 2003; Bishop et al., 2004b; Basso et al., 2007). The latter have been demonstrated with emotional Stroop (Mathews et al., 1997) and associated with deficits that are particular to the execution of attentional inhibition (Fox, 1994). This impaired cognitive control over threat-related distractors has also been supported by fMRI studies (Bishop et al., 2004a).

The effects of co-morbid anxiety in MDD have been systematically investigated in only a few studies. It has been suggested that executive dysfunction and psychomotor slowing are specific to the depressed group with co-morbid anxiety (Basso et al., 2007). The latter group also displayed more impaired scores than both "pure" MDD and HC. The limitations of this study are mainly the reliability of patient diagnoses; the data were collected retrospectively from available records, and the diagnoses lacked a structural diagnostic interview. The patient sample also consisted entirely of inpatients, and the literature suggests that inpatients are more severely impaired than outpatients (Burt et al., 1995; Christensen et al., 1997; Veiel, 1997). Corroborating these results, a recent study concluded that the strongest predictor of poor cognitive performance in depression was psychiatric co-morbidity (Baune et al., 2009). The co-morbid group showed decreased cognitive performance in visuospatial and language domains and in total score on the repeatable battery for the assessment of neuropsychological status (RBANS) in relation to the pure depression group. They found no relevant differences in mean scores for the depressed group in the study and the depressed group in the RBANS validation study. However, the inclusion of a healthy control group would give an indication of how the clinical groups differ compared to a healthy population.

On the other hand, in a review Levin et al. (2007) states that when the noise introduced by unassessed co-morbid anxiety is successfully addressed, executive function impairments specific to depression can account for many of the cognitive deficits identified. Similarly, within the context of a population based sample it was found that the subgroups of MDD and MDD with co-morbid Axis I disorder did not differ on any of the cognitive measures assessed, and conclude that psychiatric co-morbidity may not aggravate cognitive functioning among depressed young adults (Castaneda et al., 2009).

While a substantial number of patients with MDD have comorbid anxiety that possibly contributes to cognitive impairments, most patients with MDD also experience recurrent episodes. Research on the possible effects of number of episodes on cognition has yielded inconsistent results regarding whether number of recurrent depressive episodes confounds with increased cognitive impairment or not (Veiel, 1997; Basso and Bornstein, 1999; Stordal et al., 2004). In light of the previous limited research, inconsistent results and clinical importance, there is a vital need for further research to address these issues.

Although a considerable body of research documents that children of depressed parents exhibit higher rates of mood disorders than comparison groups (see Klimes-Dougan et al., 2006), there are only a few studies that have been directed toward examining neurocognitive functioning in this risk group. One of few studies that examined family history and executive functions is KlimesDougan et al. (2006) who studied neuropsychological functioning in offspring of parents with a history of mood disorders and found deficits in, e.g., executive functioning in BPD offspring compared to offspring of well mothers. Deficits were not found for children of MDD mothers. Still, heritability is estimated as high as $70 \%$. Characteristics that have generally been shown to predict a larger increase in risk to their relatives are, e.g., recurrent episodes (Sullivan et al., 2000) and earlier age at onset (see review by Levinson et al., 2003).

The main aim of this study was to investigate basic components of cognitive control functions in patients with MDD with or without co-morbid anxiety disorders, and compare to a healthy control group. Consistent with prior research regarding neurocognition in affective disorders, we expected both patient groups to perform worse than the $\mathrm{HC}$, but that the MDDA group would perform worse than the MDD without anxiety. Possible effect of number of depressive episodes and age of onset first depressive episode was also investigated.

\section{MATERIALS AND METHODS}

The study was approved by the Regional Committee for Medical Research Ethics, Norwegian Social Science Data Services (NSD) and adhered to the Helsinki Convention.

Written informed consent was obtained after the participants had been provided with a complete description of the study. 


\section{PARTICIPANTS}

A total of 165 subjects were included in the study. From this sample, 61 fulfilled the criteria for current primary non-psychotic unipolar major depression (MDD), according to the Structured Clinical Interview for Diagnostic and Statistical Manual of Mental Disorders for Axis I (DSM-IV; APA, 1994). Of these 24 subjects fulfilled the MDD criteria with co-morbid anxiety disorder, whereas 37 had no co-morbid anxiety disorder.

The distribution of the anxiety subtypes for the MDDA group was: $\mathrm{GAD}=3, \mathrm{SP}=13, \mathrm{OCD}=4, \mathrm{PTSD}=2$, panic with agoraphobia $=6$, panic without agoraphobia $=4$, specific phobia $=3$, anxiety NOS $=2$. Fourteen patients had one anxiety diagnosis, and 10 patients had two or three anxiety disorders.

The proportion of patients medicated in each group was: MDD $($ SSRI $=12$, SNRI $=1)$ and MDDA (SSRI $=9$, SNRI $=2)$. In the MDD group, 24 were unmedicated and there were 13 unmedicated in the MDDA group. The HC group was unmedicated.

The number of depressive episodes was distributed as follows for the MDD group: 1 episode (the current one) $=20,2=5,3=3$, $4=2,5=2,6=1,10=2,40=2$. For the MDDA group: 1 episode (the current one) $=12,2=3,3=2,4=1,6=1,10=1,15=1$, above 50 episodes $=3$. The MDD group had one with dysthymia (i.e., "double-depression"), and the MDDA group had 2 with dysthymia (i.e., "double-depression"). All the subjects in the MDD and MDDA group had a non-melancholic subtype. The distribution of family history of depression versus no history, were as follows: HC (77/15), MDD (4/33), and MDDA (6/18).

The patients were recruited from psychiatric clinics for outpatients in the Oslo and Trondheim area. Inclusion criteria were: to be fluent in Norwegian, and to be taking no medication other than SSRI/SNRI. The subjects were required to be medication-fasting the day of testing. General exclusion criteria were: a history of neurological disorders, bipolar, psychosis, drug/alcohol-abuse including non-prescription OTC drugs. Two participants were excluded due to scaled score $\leq 4$ on the Wechsler Adult Intelligence Scale Third edition (WAIS-III) subtest Similarities. For comparison, 91 healthy controls (HC) were selected from an original sample of 125 subjects. They were recruited via newspaper ads, posters and through various private companies in the Oslo region. They were screened for psychopathology using SCID-I and SCID-II. Thirty-three subjects were excluded from the original sample of 125 due to the following criteria: brain injury exceeding $30 \mathrm{~min}$. loss of consciousness $(n=2), \mathrm{BDI} \geq 10(n=9)$, weekly alcohol intake $>15$ IU $(n=1)$, daily use of drugs $(n=1)$, and personality disorder (according to DSM-IV; SCID-II; $n=2$ ). Furthermore, 18 participants were excluded who had had one or more depressive episodes in the past.

\section{CLINICAL EVALUATION}

Clinical and diagnostic assessment was made in accordance with the structured clinical interviews for DSM-IV criteria (SCID). Both SCID-I and SCID-II were used by trained professional clinical psychologists. In addition, the rating was blindly repeated using the original audio taped interviews, by an external experienced clinical psychologist who was unaware as to whether the participants where HC, MDD, or MDDA (validated by one of the co-authors: TCS).
The Beck Depression Inventory (BDI-II; Beck et al., 1961) and The Beck Anxiety Inventory (BAI; Beck and Steer, 1988) were filled out by the participants to measure severity of depression and anxiety symptoms. General level of functioning were screened using Global Assessment of Functions Scale for symptom and function (GAF-S and GAF-F; DSM-IV).

General medical and psychiatric background, demographic information, past episodes of recurrent major depression and family medical and psychiatric history were obtained using a semi-structured interview developed by the first author (Lyche, 2006) based on the diagnostic interview for genetic studies (DIGS; Nurnberger et al., 1994) and a semi-structured screening instrument developed by Biringer (2005), University of Bergen. Education level was classified by means of The International Standard Classification of Education (ISCED; UNESCO, 1997).

\section{GENERAL COGNITIVE MEASURES}

General cognitive functioning was estimated from the mean of two subtests from the WAIS-III: picture completion (PC) andsimilarities (SI) (Wechsler, 1999).

\section{NEUROPSYCHOLOGICAL ASSESSMENT}

Three subtests from the Cambridge Neuropsychological Test Automated Battery (CANTAB) which measure executive control functions according to Miyake et al. (2000) model were administered. In addition; a motor screening test (MOT) was administered in the beginning of the test session.

The neuropsychological tests were administered by a clinical psychologist trained in standardized assessment, or by an experienced test technician.

\section{Cambridge Neuropsychological Test Automated Battery}

The Cambridge Neuropsychological Test Automated Battery (CANTAB, 2006) is comprised of computerized neuropsychological tests that examine various areas of cognitive function. The CANTAB tests are simple, computerized, non-linguistic and culturally blind, and allow us to break down executive control functions into cognitive components in order to define more readily which functions are impaired or spared. Three subtests were used in this study; intra-extra dimensional (ID/ED), spatial working memory (SWM), and stop signal task (SST), which correspond to the three functional areas according to Miyake et al. (2000) non-unitary model of executive functions.

Shifting. The ID/ED set-shift task is a test of rule acquisition and reversal. The task assesses the ability to maintain attention to different examples within a stimulus dimension (ID stages), and the ability to shift attention to a previously irrelevant stimulus dimension (ED shifts) across nine stages. Subjects proceed to the next stage when a criterion of six consecutive correct responses has been reached.

The two performance variables of interest are the number of stages completed, and the total error score adjusted for whether the entire task is completed (Kaplan et al., 2006).

Inhibition. The SST is a classic stop signal response inhibition test (Logan et al., 1984, 1997; Osman et al., 1986). It uses staircase functions to generate an estimate of stop signal reaction time. The test gives 
a measure of an individual's ability to inhibit a prepotent response. The term "response inhibition" refers to cognitive processes enabling executive control over prepotent responses in accordance with changing situational demands. The main outcome variable of interest for our purpose is the stop signal reaction time (SSRT), which is an estimate of the subject's response time to the stop signal, i.e., the time it takes to react to the stop signal by inhibiting the prepotent response to the go signal (i.e., the time required to inhibit a response after it has been initiated). SSRT is estimated by subtracting stop signal delay (SSD) from mean Go signal reaction time (RTonGT).

Updating. Spatial working memory is a test of the subject's ability to retain spatial information and to manipulate remembered items in working memory. It is a self-ordered task which requires subjects to search for hidden "tokens" within a spatial array of colored boxes. By touching the boxes and using a process of elimination, the subject should find one blue "token" in each of a number of boxes, and then use them to fill up an empty column on the right hand side of the screen. The number of boxes is gradually increased until it is necessary to search a total of eight boxes. The color and position of the boxes used are changed from trial to trial in order to discourage the use of stereotyped search strategies. The rationale for this task and its implementation has been described previously in some detail (Owen et al., 1990). Outcome measures of interest are a "between" errors score, which is calculated when a subject returns to a square where a token was already found in a previous trial. This assesses the accuracy of working memory, as a strategy measure is derived; i.e., a subject's ability to adopt a systematic search strategy: a lower strategy score reflects more efficient task performance on SWM (Owen et al., 1990; Weiland-Fiedler et al., 2004).

\section{PSYCHOMOTOR SPEED}

In addition to the main variable of inhibition on the SST task, an outcome measure of reaction time on Go trials (GoRT), an indicator of psychomotor speed, was included.

\section{DATA ANALYSIS}

Data analysis was completed by means of SPSS version 16.0 (SPSS Inc., Chicago, IL, USA). Multivariate analysis of covariance (MANCOVA) was first used to compare the patient groups (MDD and MDDA) and the HC on the combined score of the six dependent variables measured at the individual level: ID/ED stages completed, ID/ED total errors adjusted, SWM strategy, SWM between errors, SST RT on Go trials, and SST SSRT, while simultaneously controlling for age, sex, and education level. Follow-up univariate analysis of covariance (ANCOVA) was then performed to detect which of the six dependent variables the three groups differed significantly from each other in mean scores. Finally, ANCOVA was also used to compare the MDD group and the MDDA group on the six dependent variables controlling for the number of earlier depressive episodes and age of onset of first depressive episode.

\section{RESULTS}

A series of one-way analyses of variance (ANOVAs) and Chi-square tests was conducted to compare the four groups across the demographic variables, followed by post hoc tests with Bonferroni corrections to determine which groups differed (Table $\mathbf{1}$ ).
Table 1 | Descriptive statistics for demographic variables, clinical characteristics, and general cognitive functioning (estimated from the WAIS-III subtests Similarities and Picture Completion) for patients with MDD, MDDA, and healthy comparison subjects.

\begin{tabular}{|c|c|c|c|c|c|c|}
\hline & \multicolumn{2}{|c|}{$\operatorname{MDD}(n=37)$} & \multicolumn{2}{|c|}{ MDDA $(n=24)$} & \multicolumn{2}{|c|}{$\begin{array}{l}\text { Healthy } \\
\text { controls } \\
(n=91)\end{array}$} \\
\hline & $M$ & SD & $M$ & SD & $M$ & SD \\
\hline Age (years) & 44.2 & 12.3 & 35.5 & 14.2 & 35.8 & 12.0 \\
\hline Education (years) & 15.8 & 3.2 & 13.5 & 2.3 & 16.4 & 2.5 \\
\hline $\mathrm{BDI}$ & 21.4 & 11.1 & 25.4 & 7.4 & 2.1 & 2.7 \\
\hline BAl & 11.6 & 8.2 & 18.7 & 6.7 & 2.1 & 2.5 \\
\hline Symptom-GAF & 60.2 & 12.1 & 53.8 & 8.5 & 95.6 & 7.3 \\
\hline Function-GAF & 63.6 & 13.8 & 55.2 & 10.1 & 96.7 & 5.6 \\
\hline $\begin{array}{l}\text { General cognitive } \\
\text { functioning }\end{array}$ & 11.1 & 2.1 & 9.3 & 2.6 & 12.1 & 2.4 \\
\hline \multirow{2}{*}{$\begin{array}{l}\text { Age of onset first } \\
\text { MDD episode }\end{array}$} & 33.5 & 14.4 & 30.1 & 16.1 & & \\
\hline & $n$ & $\%$ & $n$ & $\%$ & $n$ & $\%$ \\
\hline \multicolumn{7}{|l|}{ GENDER } \\
\hline Male & 14 & 37.8 & 11 & 45.8 & 28 & 30.7 \\
\hline Female & 23 & 62.2 & 13 & 54.2 & 63 & 69.3 \\
\hline \multicolumn{7}{|l|}{ HANDEDNESS } \\
\hline Right & 35 & 94.6 & 24 & 100 & 8 & 42.1 \\
\hline Left & 1 & 2.7 & 0 & 0 & 11 & 57.9 \\
\hline Ambidextrous & 1 & 2.7 & 0 & 0 & 0 & 0 \\
\hline
\end{tabular}

$M D D$, current major depressive disorder; BDI, Beck depression inventory; $B A I$, Beck anxiety inventory; GAF, global assessment of functioning axis- $V$ in Diagnostic and statistical manual of mental disorders - Fourth Edition (DSM-IV).

Differences between the groups were found regarding age $[F(2,152)=6.637, p<0.01]$, education level (ISCED) $[F(2$, $152)=9.67, p<0.001]$, and general cognitive functioning (WAISMEAN $)[F(2,152)=14.055, p<0.001]$. There were no significant differences between MDD and MDDA on BDI, but MDDA had a higher score on $\mathrm{BAI}$ than $\operatorname{MDD}[F(2,151)=115.259$, $p<0.001]$.

There were no significant between-group differences in terms of gender $\left[\chi^{2}(2)=1.85, p=0.397\right]$ distribution and handedness $\left[\chi^{2}(4)=8.60, p=0.072\right]$.

The patients with MDD differed significantly from the HC group regarding age, the mean age being older for MDD. The MDDA group differed significantly from the MDD group, the mean age being younger for the former. There were no statistical differences in age between MDDA and HC.

According to estimated general cognitive functioning, the MDDA group had significantly lower mean than HC and MDD. The MDDA differed significantly from the HC group regarding education level, the former having lower mean ISCED-level.

Since educational level and estimated general cognitive functioning was correlated we did not enter the latter in the MANCOVA. 
Although there was a significant difference between the MDDA group and $\mathrm{HC}$ on the general cognitive measure, the patients nevertheless performed well within the normal range. As compared to the formal norms the performance level equals less than one-third SD from the mean.

The MANCOVA demonstrated a significant overall group difference in performance on the CANTAB tests $[F(12,284)=2370$, $p<0.01]$ Follow-up ANCOVAs and post hoc tests demonstrated that after controlling for gender, age, and education level and adjusted for multiple comparisons (Bonferroni), there were significant differences between the MDDA group and $\mathrm{HC}$ on two of the dependent variables: ID/ED total errors adjusted and SWM between errors. The MDDA group performed the tasks with more errors than the HC.

There were significant differences between the MDD group and the HC group on SST RT on Go Trials. MDD performed the task with slower psychomotor speed compared to the HC group. There were no statistical significant differences between the clinical groups on any of the other variables.

The results and mean scores for each group are presented in Table 2. Profiles of the three groups on the CANTAB variables are presented in Figure 1.

Table 2 | Neuropsychological test performance on the CANTAB variables for patients with MDD, MDDA, and healthy comparison subjects (HC). Difference in mean scores is controlled for age, gender, and education level.

\begin{tabular}{|c|c|c|c|c|c|c|c|c|c|}
\hline & \multicolumn{2}{|c|}{$\operatorname{MDD}(n=37)$} & \multicolumn{2}{|c|}{ MDDA $(n=24)$} & \multicolumn{2}{|c|}{ Healthy controls $(n=91)$} & \multicolumn{3}{|c|}{ Statistical analyses ANCOVA } \\
\hline & $M$ & SD & $M$ & SD & M & SD & $F$ & df & $p$ \\
\hline ID/ED; stages completed & 8.4 & 1.6 & 8.3 & 0.9 & 8.7 & 0.7 & 1.399 & 2 & 0.250 \\
\hline ID/ED; total errors adj. & 25.8 & 19.4 & 33.0 & 22.0 & 20.4 & 18.0 & 4.255 & 2 & $0.016^{*}$ \\
\hline SWM; strategy & 32.3 & 6.0 & 33.1 & 5.4 & 30.8 & 6.0 & 1.764 & 2 & 0.175 \\
\hline \multicolumn{10}{|l|}{ INHIBITION } \\
\hline SST; SSRT last half & 189.1 & 64.9 & 202.5 & 74.5 & 180.1 & 50.4 & 1.785 & 2 & 0.171 \\
\hline \multicolumn{10}{|l|}{ RT/PSYCHOMOTOR SPEED } \\
\hline SST; RT on GO trials & 506.1 & 140.8 & 412.3 & 82.7 & 407.7 & 123.7 & 3.701 & 2 & $0.027^{*}$ \\
\hline
\end{tabular}

${ }^{*} p<0.05$.

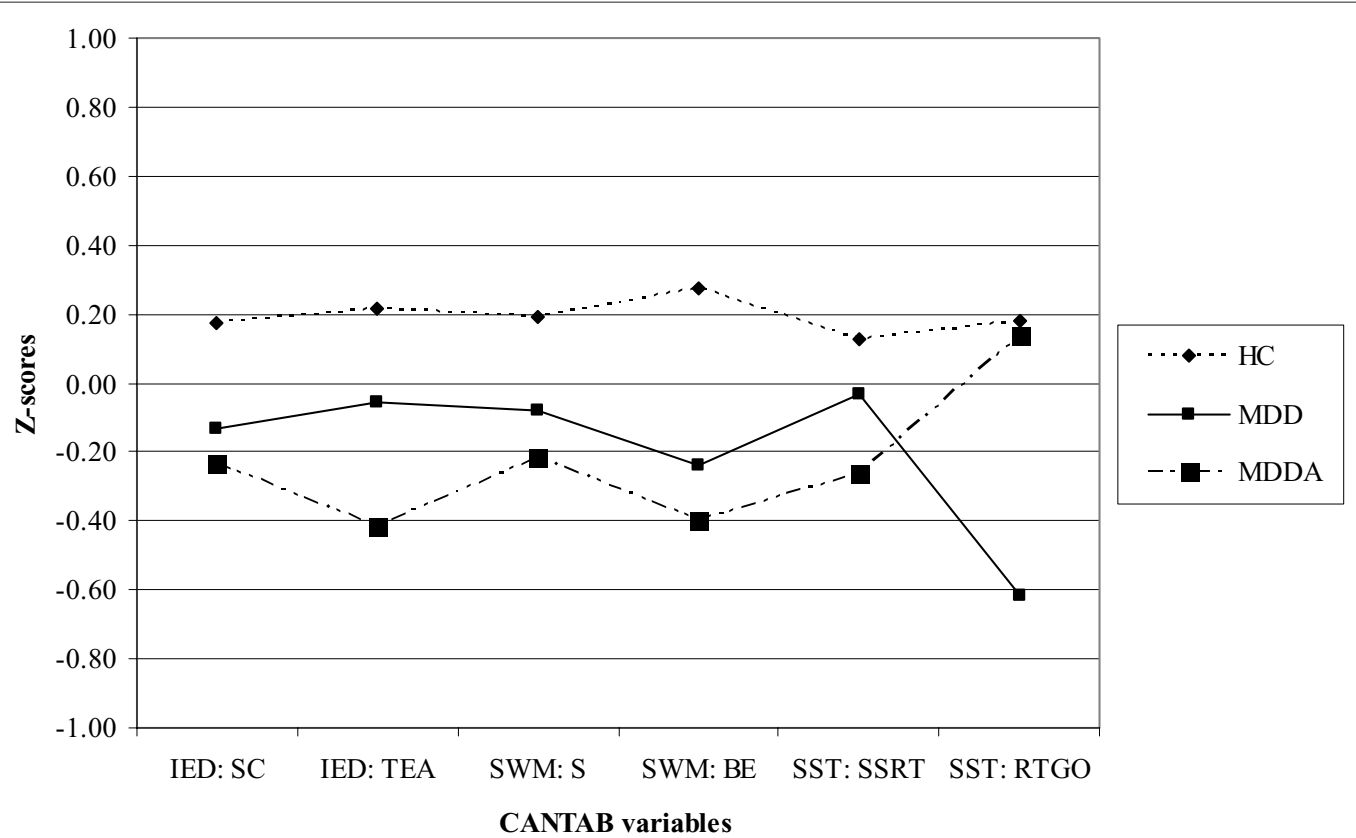

Notes: ID/ED:SC= Stages Completed, ID/ED:TEA $=$ Total Errors Adjusted, SWM:S $=$ Strategy, SWM:BE= Between Errors, SST:SSRT= Stop Signal Reaction Time, SST:RTGO=RT on Go Trials

FIGURE 1 | Profiles for the three groups on the six CANTAB variables (z-scores). 
Furthermore, we performed two ANCOVAs to test whether there was a difference between the two mood disorder patient groups (MDD and MDDA) on the CANTAB variables when we controlled for the number of depressive episodes and age of onset first depressive episode. (There are nine missing in the MDD group and four missing in the MDDA group due to difficulties in gaining accurate information about age of onset from these subjects).

The results showed that the number of episodes and age of onset was not significantly related to the dependent variables. Hence, including number of depressive episodes and age of onset as covariates in the analyses, did not alter the results, i.e., there were no significant differences in mean scores on the dependent variables between the two groups.

A significant Pearson correlation was found for the MDDA group between BAI and ID/ED; total errors adjusted $(r=0.48$, $p<0.05)$. There were no significant correlations for the SWM; between errors. No significant correlations were found between $\mathrm{BDI}$ and the two CANTAB measures.

\section{DISCUSSION}

The current data indicate that significant neuropsychological impairment in cognitive control functions corresponds with the presence of MDD and co-morbid anxiety. The MDDA group displayed significantly impaired performance with respect to working memory and set shifting compared to the HC. The MDD group did not show cognitive control dysfunctions relative to the $\mathrm{HC}$, except for displaying slowed psychomotor speed. Contrary to expectation the MDDA group did not perform worse than the MDD group. There emerged no significant differences between any of the clinical groups and HC on the ability to inhibit a prepotent response. Number of depressive episodes and age of onset for first depressive episode was not significantly related to either working memory or set shifting test performance.

The MDDA group displayed impairment in relation to the HC group on the variable assessing updating in working memory. This is an error score, and not the variable assessing the ability to adopt a systematic strategy. This indicates that it is the "pure" working memory that is affected. This is in concert with general theories of affect-cognition interactions, stating that anxiety impacts cognitive load by the depletion of central executive resources, i.e., restrictions in WM capacity (Eysenck and Calvo, 1992; Shackman et al., 2006).

Negative mood and rumination require generally more attention than neutral and positive stimuli (Dolcos and McCarthy, 2006; Van Dillen and Koole, 2007). Strong negative stimuli (as in major depression) may trigger more mood-congruent processing and thus employ more WM capacity. This interactive relationship between WM load and emotional intensity of negative stimuli were examined by Van Dillen and Koole (2007). Participants reported attenuated negative moods in WM trials with high demands, suggesting that negative mood and WM capacity interact. Since we found no significant correlation between symptom severity (BAI or BDI) for the MDDA group on the working memory variable, this may indicate that it is the diagnostic validity of the clinical ratings of syndrome MDD and co-morbid anxiety disorder that is reflected in the WM impairment. This adds data to the ongoing discussion and research about whether cognitive dysfunctions in
WM in depression and anxiety represent symptom or syndrome factors, or an interaction of both with situational stress as a mediating factor (Eysenck and Calvo, 1992; Chong, 2003; Murray and Janelle, 2003).

The ID/ED results indicate that the MDDA group showed more total errors and thus were less able than HC to learn to shift or switch attention to new previously irrelevant exemplars of stimuli or mental sets. Symptom severity on BAI, but not BDI, showed a significant correlation with set shifting. This indicates that selfreported anxiety symptom severity and the set-shift function are linked in the MDDA group; this relates to the specific roles of symptom and syndrome anxiety in executive control functions.

In contrast to Basso et al. (2007), we found no significant psychomotor slowing in the MDDA group. However, in the MDD group showed reduced psychomotor speed compared to HC. The research field has published frequent findings of psychomotor slowing in both MDD and in MDDA, but to our knowledge only a few studies (Dutke and Stöber, 2001) have found more rapid psychomotor speed in anxiety. There are numerous theories stating that the presence of anxiety yields slower processing due to an obsessive or ruminative approach to testing (Basso et al., 2007). Moreover, the profile and nature of cognitive dysfunction is hypothesized to depend upon anxiety subtype, and the majority of studies have focused mainly on OCD.

Anxiety patients often exhibit a fear of new or unfamiliar situations. Therefore the experimental situation itself is often sufficiently threatening to arouse a significantly greater amount of manifest anxiety in the anxiety group than in than the other two groups with depression. One may wonder if this creates a drive or motivation that can be measured as increased psychomotor speed. It may be hypothesized that in this study, it is the depression component that contributes to the deficits in speed, and that the lack of psychomotor deficit in the MDDA group was due to counterbalance by two inverse tendencies, or to a competition between psychomotor retardation and "acceleration."

Number of earlier depressive episodes in the MDD and MDDA group was not significantly associated with the dependent variables. This result contradicts research findings where number of depressive episodes seems to have a cumulative effect on cognitive control impairment in MDD (Vanderhasselt and De Raedt, 2009). One of the main hypotheses is that each repeated depressive episode "leaves a mark" in the brain (Sheline, 2000). Stordal et al. (2005) found no impairment in executive functions in about half the patients with recurrent MDD, although the depressed patients with executive function impairments were the ones with more episodes than the patients without such impairments. In line with our present results, other studies have found no association between number of episodes and performance on tests that tap executive functions (Reischies and Neu, 2000; Grant et al., 2001).

Regarding the notion that the MDDA group represents an additive effect in severity, studies by Purcell et al. (1997), Elliott et al. (1996), and Beats et al. (1996) show that both inpatients with a more severe history of depression and hospitalization, and elderly patients may exhibit more evident set-shift deficits than do younger/middle-aged outpatients (Purcell et al., 1997). One reason why the research field has yielded different results may be that to our knowledge, the previous research concerning MDD with 
co-morbid anxiety was based mostly on inpatient samples (i.e., Basso et al., 2007); the literature concerning neuropsychological function in depression suggests that inpatients are more severely impaired than outpatients (Burt et al., 1995; Veiel, 1997).

It seems that the "error component" is mutual for both the WM and the ID/ED tasks, and this is where MDDA patients are dysfunctional. Theories state that ACC is involved in, i.e., error detection (Bush et al., 2000). It has frequently been hypothesized to make critical contributions to the function of neural systems involved in the executive control of cognition (Carter et al., 1999). It may be hypothesized that ACC may be one area in common for the co-morbid group's error component in performance on both SWM and ID/ED.

There are some potential limitations in this study. In light of the present findings additional research is needed to further address and possibly replicate current or previous findings on the effects of co-morbid anxiety disorders on executive control functions in patients with MDD. It would be advantageous to use larger samples, differentiated patient diagnoses and multiple tests to assess cognitive control functions. Possible effects of medication may also be a factor. Even though most of the patients in the two clinical groups were medication-fasting the day of testing, these subjects cannot be considered medication-free in regard to SSRI/SNRI. However, the proportion of subjects in each group with versus without medication may be considered fairly representative of the clinical

\section{REFERENCES}

APA. (1994). Diagnostic and Statistical Manual of Mental Disorders, DSM-IV. Washington, DC:American Psychiatric Association.

Asmundson, G. J., Stein, M. B., Larsen, D. K., and Walker, J. R. (1994). Neurocognitive function in panic disorder and social phobia patients. Anxiety 1, 201-207.

Austin, M. P., Mitchell, P., and Goodwin, G. M. (2001). Cognitive deficits in depression: possible implications for functional neuropathology. $\mathrm{Br}$. J. Psychiatry 178, 200-206.

Basso, M. R., and Bornstein, R. A. (1999). Relative memory deficits in recurrent versus first-episode major depression on a word list learning-task. Neuropsychology 13, 557-563.

Basso, M. R., Lowery, N., Ghormley, C., Combs, D., Purdie, R., Neel, J., Davis, M., and Bornstein, R. (2007). Comorbid anxiety corresponds with neuropsychological dysfunction in unipolar depression. Cogn. Neuropsychiatry 12, 437-456.

Baune, B. T., McAfoose, J., Leach, G., Quirk, F., and Mitchell, D. (2009). Impact of psychiatric and medical comorbidity on cognitive function in depression. Psychiatry Clin. Neurosci. 63, 392-400.

Beats, B. C., Sahakian, B. J., and Levy, R. (1996). Cognitive performance in tests sensitive to frontal lobe dysfunction in the elderly depressed. Psychol. Med. 26, 591-603.
Beck, A. T., and Steer, R. A. (1988). Beck Anxiety Inventory (BAI). San Antonio: Harcourt Assessment, Inc.

Beck, A. T., Steer, R. A., and Brown, G. K. (1961). Beck Depression Inventory (BDI-II), 2nd Edn. San Antonio: Harcourt Assessment, Inc.

Biringer, E. (2005). Semi-structured Screening Instrument for Recurrent Depressive Episodes and Length of Episodes. Doctoral thesis, Department of Psychiatry, Faculty of Medicine, University of Bergen, Norway.

Bishop, S., Duncan, J., Brett, M., and Lawrence, A. D. (2004a). Prefrontal cortical function and anxiety: controlling attention to threat-related stimuli. Nat. Neurosci. 7, 184-188.

Bishop, S. J., Duncan, J., and Lawrence, A. D. (2004b). State anxiety modulation of the amygdala response to unattended threat-related stimuli. J. Neurosci. 24, 10364-10368.

Broadbent, D. E., Broadbent, M. H., and Jones, J. L. (1986). Performance correlates of self-reported cognitive failure and of obsessionality. Br. J. Clin. Psychol. 25 (Pt 4), 285-299.

Burt, D. B., Zembar, M. J., and Niederehe, G. (1995). Depression and memory impairment: a meta-analysis of the association, its pattern, and specificity. Psychol. Bull. 117, 285-305.

Bush, G., Luu, P., and Posner, M. I. (2000). Cognitive and emotional influences in anterior cingulate cortex. Trends Cogn. Sci. 4, 215-222.

population with a larger proportion of medicated subjects in the MDDA group. Research suggests both that modern antidepressants do not have deleterious effects on cognitive test performance, and that that unmedicated patients may show more impaired attention functions compared to patients on medication (Den Hartog et al., 2003). Some studies also suggest that antidepressants may improve cognitive functions, including attention, in MDD patients (Fergusson et al., 2003), and that the effects of medication may be different on MDD from those with MDDA (Herrera-Guzmàn et al., 2009). Furthermore, functional imaging should be included to provide a greater understanding of the associated neurobiological correlates of cognitive control functions.

In sum, the data suggest that the presence of co-morbid anxiety in MDD results in impairment of the basic cognitive control functions Updating and Shifting, whereas patients with MDD displayed no cognitive control impairment, but reduced psychomotor speed.

\section{ACKNOWLEDGMENTS}

The study was supported by a grant from the Department of Psychology, University of Oslo to Pia Lyche, and a grant from the Research Council of Norway to Nils Inge Landrø. We wish to thank research assistants Martin Aker, Anne Marie Hoel, and Monika Rutle for assisting us with the data collection. We also thank Jan Egil Nordvik and Marcus Sneve Handal.

Cameron, O. G., Abelson, J. L., and Young, E. A. (2004). Anxious and depressive disorders and their comorbidity: effect on central nervous system noradrenergic function. Biol. Psychiatry 56, 875-883.

Carter, C. S., Botvinick, M.M., and Cohen, J. D. (1999). The contribution of the anterior cingulate cortex to executive processes in cognition. Rev. Neurosci. 10, 49-57.

Castaneda,A.E., Marttunen, M., Suvisaari, J., Perala, J., Saarni, S. I., Aalto-Setala, T., Aro, H., Lonnqvist, J., and TuulioHenriksson, A. (2009). The effect of psychiatric co-morbidity on cognitive functioning in a population-based sample of depressed young adults. Psychol. Med. 40, 29-39.

Chong, J. L. Y. (2003). Anxiety and Working Memory: An Investigation and Reconceptualisation of the Processing Efficiency Theory. Thesis for the degree of Master of Psychology and Doctor of Philosophy, The University of Western Australia, Australia.

Christensen, H., Griffiths, K., Mackinnon, A., and Jacomb, P. (1997). A quantitative review of cognitive deficits in depression and Alzheimer-type dementia. J. Int. Neuropsychol. Soc. 3, 631-651.

Cohen, L. J., Hollander, E., DeCaria, C. M., Stein, D. J., Simeon, D., Liebowitz, M. R., and Aronowitz, B. R. (1996) Specificity of neuropsychological impairment in obsessive-compulsive disorder: a comparison with social phobic and normal control subjects. J. Neuropsychiatry Clin. Neurosci. 8, 82-85.

Daban, C., Colom, F., Sanchez-Moreno, J., Garcia-Amador, M., and Vieta, E. (2006). Clinical correlates of firstepisode polarity in bipolar disorder. Compr. Psychiatry 47, 433-437.

Danckwerts, A., and Leathem, J. (2003). Questioning the link between PTSD and cognitive dysfunction. Neuropsychol. Rev. 13, 221-235.

Den Hartog, H. M., Derix, M. M. A., van Bemmel, A. L., Kremer, B., and Jolles, J. (2003). Cognitive functioning in young and middle-aged unmedicated out-patients with major depression: testing the effort and cognitive speed hypotheses. Psychol. Med. 33, 1443-1451.

Denckla, M. B. (1996). "A theory and model of executive function: a neuropsychological perspective," in Attention, Memory, and Executive Function, eds G. R. Lyon and N. A. Krasnegor (Baltimore, MD: Brookes), 263-278.

Dennis, T. A., and Chen, C. C. (2007). Neurophysiological mechanisms in the emotional modulation of attention: the interplay between threat sensitivity and attentional control. Biol. Psychol. 76, 1-10.

Dolcos, F., and McCarthy, G. (2006). Brain systems mediating cognitive interference by emotional distraction. $J$. Neurosci. 26, 2072-2079. 
Dutke, S., and Stöber, J. (2001). Test anxiety, working memory and cognitive performance: supportive effects of sequential demands. Cogn. Emot. 15, 381-389.

Egeland, J., Lund, A., Landro, N. I., Rund, B. R., Sundet, K., Asbjornsen, A., Mjellem, N., Roness, A., and Stordal, K. I. (2005). Cortisol level predicts executive and memory function in depression, symptom level predicts psychomotor speed. Acta Psychiatr. Scand. 112, 434-441.

Elliott, R., Sahakian, B. J., McKay, A. P., Herrod, J.J., Robbins, T.W., and Paykel, E. S. (1996). Neuropsychological impairments in unipolar depression: the influence of perceived failure on subsequent performance. Psychol. Med. 26, 975-989.

Eysenck, M. W., and Calvo., M. G. (1992). Anxiety and performance: the processing efficiency theory. Cogn. Emot. 6, 409-434.

Fergusson, D. M., Goodwin, R. D., and Horwood, L. J. (2003). Major depression and cigarette smoking: results of a 21-year longitudinal study. Psychol. Med. 33, 1357-1367.

Fox, E. (1994). Attentional bias in anxiety: a defective inhibition hypothesis. Cogn. Emot. 8, 165-195.

Grant, M. M., Thase, M. E., and Sweeney, J. A. (2001). Cognitive disturbance in outpatient depressed younger adults: evidence of modest impairment. Biol. Psychiatry 50, 35-43.

Gruber, S., Rathgeber, K., Braunig, P., and Gauggel, S. (2007). Stability and course of neuropsychological deficits in manic and depressed bipolar patients compared to patients with major depression. J. Affect. Disord. 104, 61-71.

Herrera-Guzman, I., Gudayol-Ferre, E., Jarne-Esparcia, A., Herrera-Abarca, J. E., Herrera-Guzman, D., PeroCebollero, M., and Guardia-Olmos, J. (2009). Comorbidity of anxiety disorders in major depressive disorder. A clinical trial to evaluate neuropsychological deficit. Eur. J. Psychiatry 23, 5-18.

Kaplan, J. S., Erickson, K., Luckenbaugh, D. A., Weiland-Fiedler, P., Geraci, M., Sahakian, B. J., Charney, D., Drevets, W. C., and Neumeister, A. (2006). Differential performance on tasks of affective processing and decisionmaking in patients with panic disorder and panic disorder with comorbid Major Depressive Disorder. J. Affect. Disord. 95, 165-171.

Kindermann, S. S., and Brown, G. G. (1997). Depression and memory in the elderly: a meta-analysis. J. Clin. Exp. Neuropsychol. 19, 625-642.

Klimes-Dougan, B., Ronsaville, D., Wiggs, E. A., and Martinez, P. E. (2006).
Neuropsychological functioning in adolescent children of mothers with a history of bipolar or major depressive disorders. Biol. Psychiatry 60, 957-965.

Landrø, N. I., Stiles, T. C., and Sletvold, H. (2001). Neuropsychological function in nonpsychotic unipolar major depression. Neuropsychiatry Neuropsychol. Behav. Neurol. 14, 233-240.

Lautenbacher, S., Spernal, J., and Krieg, J. (2002). Divided and selective attention in panic disorder. A comparative study of patients with panic disorder, major depression and healthy controls. Eur. Arch. Psychiatry Clin. Neurosci. 252, 210-213.

Leonardo, E. D., and Hen, R. (2006). Genetics of affective and anxiety disorders. Annu. Rev. Psychol. 57, 117-137.

Levin, R. L., Heller, W., Mohanty, A., Herrington, J. D., and Miller, G. A. (2007). Cognitive deficits in depression and functional specificity of regional brain activity. Cogn. Ther. Res. 31, 211-233.

Levinson, D. F., Zubenko, G. S., Crowe, R. R., DePaulo, R. J., Scheftner, W. S., Weissman, M. M., Holmans, P., Zubenko, W. N., Boutelle, S., MurphyEberenz, K., MacKinnon, D., McInnis, M. G., Marta, D. H., Adams, P., Sassoon, S., Knowles, J. A., Thomas, J., and Chellis, J. (2003). Genetics of recurrent early-onset depression (GenRED): design and preliminary clinical characteristics of a repository sample for genetic linkage studies. Am. J. Med. Genet. B Neuropsychiatr. Genet. 119B, 118-130.

Logan, G. D., Cowan, W. B., and Davis, K. A. (1984). On the ability to inhibit simple and choice reaction time responses: a model and a method. J. Exp. Psychol: Hum. Percept. Perform. 10, 276-291.

Logan, G. D., Schachar, R., and Tannock, R. (1997). Impulsivity and inhibitory control. Psychol. Sci. 8, 60-64.

Ludewig, S., Paulus, M. P., Ludewig, K., and Vollenweider, F. X. (2003). Decision-making strategies by panic disorder subjects are more sensitive to errors. J. Affect. Disord. 76, 183-189.

Lyche, P. L. (2006). Semi-structured Interview. Oslo: Department of Psychology, University of Oslo.

MacLeod, C., Rutherford, E., Campbell, L., Ebsworthy, G., and Holker, L. (2002). Selective attention and emotional vulnerability: assessing the causal basis of their association through the experimental manipulation of attentional bias. J. Abnorm. Psychol. 111, 107-123.

Mathews, A., Mackintosh, B., and Fulcher, E. P. (1997). Cognitive biases in anxiety and attention to threat. Trend $\operatorname{Cog} n$. Sci. 1, 340-345.

McNeely, H. E., Lau, M. A., Christensen, B. K., and Alain, C. (2005). Neurophysiological evidence of $\operatorname{cog}$ nitive inhibition anomalies in persons with major depressive disorder. Paper Presented At the 45th Annual Meeting of the Society-for-PsychophysiologicalResearch, Lisbon, Portugal.

Miyake, A., Friedman, N. P., Emerson, M. J., Witzki, A. H., Howerter, A., and Wager, T. D. (2000). The unity and diversity of executive functions and their contributions to complex "frontal lobe" tasks: a latent variable analysis. Cogn. Psychol. 41, 49-100.

Murray, N. P., and Janelle, C. M. (2003). Anxiety and performance: a visual search examination of the Processing Efficiency Theory. J. Sport. Exerc. Psychol. 25, 171-187.

Nolen-Hoeksema, S. (1991). Responses to depression and their effects on the duration of depressive episodes. $J$. Abnorm. Psychol. 100, 569-582.

Nurnberger, J. I., Blehar, M. C., Kaufmann, C. A., York-Cooler, C. Y., Simpson, S. G., Harkavy-Friedman, J. H., Severe, J. B., Malaspina, D., and Reich, T. (1994). Diagnostic interview for genetic studies. Rationale, unique features and training. Arch. Gen. Psychiatry 51, 849-859.

Ochsner, K. N., and Gross, J. J. (2005). The cognitive control of emotion. Trend Cogn. Sci. 9, 242-249.

Olley, A., Malhi, G., and Sachdev, P. (2007). Memory and executive functioning in obsessive-compulsive disorder: a selective review. J. Affect. Disord. 104, 15-23.

Osman, A., Kornblum, S., and Meyer, D. E. (1986). The point of no return in choice reaction time: controlled and ballistic stages of response preparation. J. Exp. Psychol: Hum. Percept. Perform. 12, 243-258.

Owen, A. M., Downes, J. J., Sahakian, B. J., Polkey, C.E., and Robbins, T.W. (1990). Planning and spatial working memory following frontal lobe lesions in man. Neuropsychologia 28, 1021-1034.

Perez-Edgar, K., Fox, N. A., Cohn, J. F., and Kovacs, M. (2006). Behavioral and electrophysiological markers of selective attention in children of parents with a history of depression. Biol. Psychiatry 60, 1131-1138.

Philips, L. H. (1997). "Do "frontal tests" measure executive function? Issues of assessment and evidence from fluency tests," in Methodology of Frontal and Executive Function, ed. P. Rabbit (Hove: Psychology Press), 191-213.

Porter, R. J., Bourke, C., and Gallagher, P. (2007). Neuropsychological impairment in major depression: its nature, origin and clinical significance. Aust. NZ J. Psychiatry 41, 115-128.
Purcell, R., Maruff, P., Kyrios, M., and Pantelis, C. (1997). Neuropsychological function in young patients with unipolar major depression. Psychol. Med. 27, 1277-1285.

Rabbit, P. (1997). "Introduction: methodologies and models in the study of executive function," in Methodology of Frontal and Executive Function, ed. P. Rabbit (Hove: Psychology Press), 1-38.

Reischies, F. M., and Neu, P. (2000). Comorbidity of mild cognitive disorder and depression - a neuropsychological analysis. Eur. Arch. Psychiatry Clin. Neurosci. 250, 186-193.

Rogers, M. A., Kasai, K., Koji, M., Fukuda, R., Iwanami, A., Nakagome, K., Fukuda, M., and Kato, N. (2004). Executive and prefrontal dysfunction in unipolar depression: a review of neuropsychological and imaging evidence. Neurosci. Res. 50, 1-11.

Shackman, A. J., Sarinopoulos, I., Maxwell, J. S., Pizzagalli, D. A., Lavric, A., and Davidson, R. J. (2006). Anxiety selectively disrupts visuospatial working memory. Emotion 6, 40-61.

Sheline, Y. I. (2000). 3D MRI studies of neuroanatomic changes in unipolar major depression: the role of stress and medical comorbidity. Biol. Psychiatry $48,791-800$.

Stordal, K. I., Lundervold, A. J., Egeland, J., Mykletun, A., Asbjornsen, A., Landro, N. I., Roness, A., Rund, B. R., Sundet, K., Oedegaard, K. J., and Lund, A. (2004). Impairment across executive functions in recurrent major depression. Nord. J. Psychiatry 58, 41-47.

Stordal, K. I., Lundervold, A. J., Mykletun, A., Asbjornsen, A., Biringer, E., Egeland, J., Hammar, A., Landro, N. I., Roness, A., Rund, B. R., Sundet, K., and Lund, A. (2005). Frequency and characteristics of recurrent major depressed patients with unimpaired executive functions. World J. Biol. Psychiatry 6, 36-44.

Sullivan, P. F., Neale, M. C., and Kendler, K. S. (2000). Genetic epidemiology of major depression: review and meta-analysis. Am. J. Psychiatry 157, 1552-1572.

The Cambridge Neuropsychological Test Automated Battery (CANTAB). (2006). Cambridge: Cambridge Cognition Limited.

UNESCO. (1997). International Standard Classification of Education. Available at: http://www.unesco.org/education/information/nfsunesco/doc/ isced_1997.htm

Van Dillen, L. F., and Koole, S. L. (2007). Clearing the mind: a working memory model of distraction from negative mood. Emotion 7, 715-723.

Vanderhasselt, M. A., and De Raedt, R. (2009). Impairments in cognitive 
control persist during remission from depression and are related to the number of past episodes: an event related potentials study. Biol. Psychol. 81, 169-176.

Vasterling, J. J., Rogers, C., and Kaplan, E. (2000). Qualitative block design analysis in posttraumatic stress disorder. Assessment 7, 217-226.

Veiel, H. O. (1997). A preliminary profile of neuropsychological deficits associated with major depression. J. Clin. Exp. Neuropsychol. 19, 587-603.

Wechsler, D. (1999). Wechsler Adult Intelligence Scale, 3rd Edn. Stockholm:
The Psychological Corporation (Manual, Norwegian version).

Weiland-Fiedler, P., Erickson, K., Waldeck, T., Luckenbaugh, D.A., Pike, D., Bonne, O., Charney, D. S., and Neumeister, A. (2004). Evidence for continuing neuropsychological impairments in depression. J. Affect. Disord. 82, 253-258.

Wilmsmeier, A., Ohrmann, P., Suslow, T., Siegmund, A., Koelkebeck, K., Rothermundt, M., Kugel, H., Arolt, V., Bauer, J., and Pedersen, A. (2010). Neural correlates of set-shifting: decomposing executive functions in schizophrenia. J. Psychiatry Neurosci. 35, 321-329.

Conflict of Interest Statement: The authors declare that the research was conducted in the absence of any commercial or financial relationships that could be construed as a potential conflict of interest.

Received: 03 May 2010; paper pending published: 28 September 2010; accepted: 24 November 2010; published online: 21 December 2010.

Citation: Lyche P, Jonassen R, Stiles TC, Ulleberg P and Landrø NI (2010) Cognitive control functions in unipolar major depression with and without co-morbid anxiety disorder. Front. Psychiatry 1:149. doi: 10.3389/fpsyt.2010.00149

This article was submitted to Frontiers in Affective Disorders and Psychosomatic Research, a specialty of Frontiers in Psychiatry. Copyright (c) 2010 Lyche, Jonassen, Stiles, Ulleberg and Landro. This is an openaccess article subject to an exclusive license agreement between the authors and the Frontiers Research Foundation, which permits unrestricted use, distribution, and reproduction in any medium, provided the original authors and source are credited. 